\title{
Kernos
}

Revue internationale et pluridisciplinaire de religion grecque antique

19 | 2006

Varia

\section{Claude CALAME, Roger CHARTIER (éds), Identités d'auteur dans l'Antiquité et la tradition européenne}

Ioanna Papadopoulou-Belmehdi

\section{(2) OpenEdition}

\section{Journals}

Édition électronique

URL : https://journals.openedition.org/kernos/506

DOI : 10.4000/kernos.506

ISSN : 2034-7871

Éditeur

Centre international d'étude de la religion grecque antique

Édition imprimée

Date de publication : 1 janvier 2006

Pagination : 497-500

ISSN : 0776-3824

Référence électronique

Ioanna Papadopoulou-Belmehdi, « Claude calame, Roger chartier (éds), Identités d'auteur dans I'Antiquité et la tradition européenne », Kernos [En ligne], 19 | 2006, mis en ligne le 22 mars 2011, consulté le 24 août 2022. URL : http://journals.openedition.org/kernos/506 ; DOI : https://doi.org/ 10.4000/kernos.506 
différents aspects de cet « hellénisme » d'époque romaine, une problématique dont on peut mesurer l'ampleur et la complexité par exemple à la lecture de la synthèse publiée tout récemment par Paul Veyne (L'Empire gréco-romain, Paris, Seuil, 2005). Le mérite de cet ouvrage n'en reste pas moins de montrer à quel point l'utilisation de la documentation épigraphique s'avère souvent primordiale pour préciser et renouveler notre connaissance des provinces et des sociétés provinciales du monde romain hellénophone, entre le II ${ }^{\mathrm{e}} \mathrm{s}$. av. J.-C et le III $^{\mathrm{e}} \mathrm{s}$. de notre ère. L'ensemble témoigne de la variété des perspectives selon lesquelles il est possible d'aborder les formes que revêt la culture grecque dans la vie civique de cette époque, en rapport notamment avec les axes grec, oriental et romain de l'identité et avec les motivations sociales et individuelles qui expliquent le rôle joué dans les cités et les provinces par ces multiples personnages dont la documentation épigraphique nous aide à reconstruire l'action et la personnalité.

Yves Lafond

(Université de Poitiers)

Claude Calame, Roger Chartier (éds), Identités d'auteur dans l'Antiquité et la tradition européenne, Grenoble, Jérôme Millon, 2004. 1 vol. 13,5×21,5 cm, $198 \mathrm{p}$.

Né d'une journée d'échange organisée par Claude Calame et Roger Chartier, à l'EHESS, à Paris (mai 2002), ce livre reflète une démarche résolument interdisciplinaire et diachronique. La majorité des contributions dialoguent avec les propositions théoriques de Foucault à propos de la notion d'« auteur » tout en traçant des parcours qui vont de la Grèce archaïque à l'époque moderne. Chaque article sur l'antiquité est suivi d'une étude sur une époque plus récente se construisant en « interaction dialoguée » pour notre plus grand profit.

En ce qui concerne l'antiquité grecque, il était temps de contextualiser une question aussi centrale que délicate, comme celle de l'auteur, après des applications parfois trop enthousiastes des thèses de Foucault : les articles de C. Calame et de G. Nagy y contribuent de façon décisive. Si Foucault se centre sur l'aspect institutionnel et juridique de la fonction « auteur », C. Calame («Identités d'auteur à l'exemple de la Grèce classique : signatures, énonciations, citations ») développe ce que Foucault esquisse à peine : la position que l'auteur assume dans son propre discours d'une part et la manière de citer une ouvre en l'attribuant à un auteur, en insistant sur la relation entre ces deux dimensions de la représentation autoriale. Comme la procédure de la signature «traverse les limites d'ailleurs floues » des grands genres, l'A. présente un large éventail de cas de signature autoriale ou de citation d'auteurs: Hésiode et Alcman, Théognis et Timothée, Bacchylide et Pindare, Thucydide et Hérodote, Hécatée, en insistant sur les modalités de la mise en discours, notamment sur la " porosité » de la distinction entre « récit» et « discours » et la relative autonomie de la composition par rapport à l'exécution. Dans une littérature à caractère traditionnel, où les manifestations poétiques sont des véritables actes rituels et communautaires, le nom d'auteur renvoie à une identité essentiellement poétique, à une figure fondatrice et " garante " d'une pratique discursive particulière.

G. Nagy («L'aède épique en auteur : la tradition des Vies d'Homère ») propose un modèle d'historicisation de la réception de la poésie homérique. Au centre de la démonstration la tradition biographique autour d'Homère, les Vies, envisagées comme des mises en scène de la réception du poète, toujours relatives à des récitations orales, dans une grande variété d'occasions dont le festival panhellénique est l'occasion de 
première importance. Trois axes parcourent ce modèle : l'accent que toutes les traditions biographiques mettent sur la nature orale des créations d'Homère (cela passe par une enquête détaillée autour de poiein et de graphein), la mise en évidence de trois strates de traditions, trois orientations politiques dans les Vies, qui correspondent à divers modes d'appropriation d'Homère à travers le monde grec; le dépassement, à propos des Vies, de la distinction mythe/histoire: les Vies sont des mythes qui font partie de l'histoire. Ces "mythes », ainsi que la sphragis dans l'Hymne bomérique à Apollon, instaurent l'autorité d'Homère. Le mythe d'Homère « performer » de la poésie qui porte son nom est présent à chaque récitation orale et c'est cette présence « construite » qui confère à la poésie son autorité et à la récitation son caractère rituel. Dans le modèle de Nagy le mythe est le rite : une dichotomie fondatrice de l'anthropologie et de l'histoire de religions se trouve ainsi questionnée, celle du mythe (parole) versus rite (acte), montrant que la poétique archaïque s'accomode difficilement de certaines catégorisations modernes. En dialogue avec G. Nagy, Roger Chartier ( La parole ailée et sacrée ») déploie un jeu de parallèles fort intéressant, en recherchant des pratiques analogues aux XVI ${ }^{e}$ et $\mathrm{XVII}^{\mathrm{e}}$ s. en Europe : légendes biographiques, supériorité de l'oral sur l'écrit, vols textuels, le mythe du poète aveugle inspiré, la coïncidence du religieux et du festif... autant de pistes où les échos entre époques éloignées réveillent des grandes questions, toujours ouvertes : survivances, analogies, invariants?

L'article de Jesper Svenbro (« La naissance de l'auteur dans une inscription grecque ») cherche à démontrer la pertinence des thèses de Foucault sur la mort de l'auteur, à partir du jeu entre troisième et première personne dans les inscriptions gravées sur les objets ou monuments «parlants », du type "Je suis tel... untel m'a dédié ». Reprenant ses thèses bien connues depuis La parole et le marbre et Phrasikleia, sur le poiêtês et sur le rapport de l'écriture à la mort et à l'absence, l'A. voit dans l'usage de la troisième personne la conscience de la future absence de l'auteur, la première personne n'étant d'ailleurs jamais utilisée pour désigner l'auteur ou le dédicant (sauf dans le cas négativement remarquable du général Pausanias). Dans le même esprit, l'A. commente brièvement le passage du « il » au « je » dans l'œuvre des premiers historiens. La démonstration dépend étroitement du concept foucaldien de la mort de l'auteur, dont la transposition sur l'antiquité grecque exige pourtant de multiples médiations, notamment pour l'époque archaïque, comme l'illustrent fortement les contributions précédentes. Suit la réflexion d'Anne-Marie Christin ("L'énonciation écrite en Europe à l'ère de l'imprimé : de la présence du blanc à la lecture d'auteur »), entre culture grecque et tradition européenne, qui porte sur l'imaginaire occidental de l'écrit, marqué par l'invention de la typographie, de la lettre-signe, du blanc et de l'interligne en tant que «signes sémantiques visuels » offerts à l'auteur. L'imprimé ne s'est affranchi du modèle de l'oralité discursive que très lentement et c'est par l'intermédiaire de l'image que l'écrit se dissocie de l'oral. Pour comprendre ce que l'écriture, dans sa lente confrontation à l'oral, doit à la peinture, il faut certainement y introduire le visuel. D'où l'intérêt des remarques de l'A. sur la fonction-auteur dans la peinture, aspect négligé par Foucault, et discuté surtout à partir de Mallarmé, selon lequel la littérature écrite relève de l'image, où l'absence de l'auteur ne signifie nullement sa mort et d'où la fonction-auteur en sort magnifiée.

André Laks ( 'Qu'importe qui parle': sur l'anonymat platonicien et ses antécédents ») introduit au débat les philosphes, en réexaminant du point de vue de la fonction-auteur, la question de «l'anonymat platonicien», cultivé par la stratégie littéraire de la forme dialogique. Commençant par s'interroger sur la nature de la sphragis chez les présocratiques, l'A. récuse la thèse de l'égotisme dans la philosophie présocratique. "Porteurs d'une certaine universalité », les ego des philosophes préso- 
cratiques ne trancheraient pas aussi nettement avec la stratégie platonicienne du dialogue, qui vise la « dépersonnalisation du discours philosophique ». L'A. commente brièvement les tendances d'interprétation depuis l'antiquité en insistant sur la tendance moderne de privilégier la prudence dans l'exercice d'attribution de telle ou telle doctrine à Platon et sur l'importance du contexte d'énonciation. Cette désontologisation du discours philosophique est particulièrement intéressante, dans la mesure où une pareille tendance peut s'observer dans d'autres domaines, comme celui de l'historiographie, par exemple : lire les formes discursives antiques - qui appartiennent à une période où la «spécialisation » est encore en cours - comme des disciplines déjà achevées depuis l'antiquité n'est certainement pas le mode de lecture le plus fécond. Dinah Ribard ("Anonymat philosophique et exigence autoriale à 1 'époque moderne ») répond à la problématique mise en place par A. Laks en observant le mouvement inverse à l'époque moderne, exemplifié par l'accès au statut d'auteurphilosophe : la désanonymisation du discours philosophique, assumer ses opinions en tant qu'auteur-philosophe (nouveau), basculer « de l'autorité savante à l'autorialité littéraire », pour évoquer encore Foucault et souligner l'opposition de l'auteur ainsi défini à l'institution et la discipline constituée.

L'article de Christian Jacob (« La construction de l'auteur dans le savoir bibliographique antique: à propos des Deipnosophistes d'Athénée ») se fonde sur le projet d'illustrer les propositions fondamentales de Foucault sur la «fonction-auteur » et la « construction » de l'auteur essentiellement à partir de la catégorie de la littérature érudite et compilatoire de l'époque impériale, une période et un milieu où le lieu d'épanouissement de la culture classique ancienne est la bibliothèque et la pratique savante de la bibliographie. Au centre de la démonstration les Deipnosophistes d'Athénée, bel exemple de cette littérature issue d'un exercice de compilation des citations érudites, une "écriture de lecteur » qu'Athénée manipule avec talent. L'analyse se déploie à deux niveaux : les auteurs cités par Athénée, Athénée comme auteur. Même dans cet exercice de compilation, l'ouvrage est fait pour correspondre à une occasion précise, celle du banquet des érudits, qu'Athénée met soigneusement en scène, et c'est justement ce cadre social qui fournit à la construction sa cohérence. Comment être auteur d'un ouvrage composé par l'accumulation d'un grand nombre de citations? En les articulant sur la pratique intellectuelle des questions et des réponses, jeu favori des lettrés dans les banquets érudits, la technique de la citation d'autres auteurs étant soumise à cette zétesis. Athénée-auteur pratique une "écriture de lecteur », une littérature de commentaire, dont il sera un représentant talentueux. Jean-Marc Chatelain (« La définition bibliographique de l'auteur, entre reconnaissance technique et reconnaissance morale ») prolonge ce parcours par une réflexion sur l'histoire des pratiques savantes à l'époque moderne, en retrouvant dans la culture humaniste de la Renaissance la même intense activité de compilation. Au sein de cette tradition se dessine peu à peu une nouvelle figure de l'auteur, issue du travail savant d'une culture de commentaire et de catalogage du savoir constitué en bibliographie, une figure qui renvoie à la fonction-auteur par la multiplicité de ses «fonctions" au sein de ce système.

Les particularités du régime d'autorialité à Rome, esquissées avec le brio habituel de Florence Dupont («Comment devenir à Rome un poète bucolique ? Corydon, Tityre, Virgile et Pollion ») offrent un terrain de comparaison particulièrement suggestif. Auctor est «l'homme capable d'introduire une nouveauté dans l'espace collectif », le « qui primum », un titre reservé primitivement à l'espace politique. Par extension, un poète devient auctor quand il introduit un genre nouveau, tel le Virgile des Bucoliques, qui sert d'exemple principal. Mais on ne devient pas poète-auctor sans le lien 
avec le patronus, puisque «le commanditaire à Rome est le véritable facteur d'un ouvrage d'art». Suit l'article de Christian Jouhaud («'Qu'est-ce un auteur?' de l'Antiquité romaine au XvII ${ }^{e}$ siècle »), qui s'interroge sur la construction de la position d'auteur en mettant en dialogue Rome et le XVII ${ }^{\mathrm{e}}$ s. en Europe, sociétés gouvernées par les liens de patronage et de clientèle, dans une perspective qui privilégie l'interaction entre pratiques d'écriture et cadres sociaux.

La structure interactive ainsi que la qualité des contributions font de ce livre un instrument de réflexion important. Vu la référence omniprésente à Foucault (à l'exception de l'article de Gregory Nagy), les hellénistes ressentiront peut-être le manque d'une mise en évidence plus claire, en guise d'épilégomènes, des divergences latentes par rapport au modèle foucaldien. Peut-être est-ce trop demander à un ouvrage déjà riche et bien orchestré. Débat à poursuivre donc, même si «la mort du sujet » ou « la question homérique » l'ont rendu marginal et aride. Il est temps de se réconcilier avec l'idée que la personne, mythique ou réelle, de l'auteur est un trait particulièrement important du système culturel grec ancien.

Ioanna Papadopoulou

(Université libre de Bruxelles)

Micol Perfigli, Indigitamenta. Divinità funzionali e Funzionalità divina nella Religione Romana, Pisa, Edizioni ETS, 2004. 1 vol. $15,5 \times 22 \mathrm{~cm}, 308$ p. (Antropoi. Studi e materiali di Antropologia storica del mondo antico, 2). ISBN : 88-467-0977-2.

Le livre de Michol Perfigli (M.P.) ouvre à nouveau un des dossiers parmi les plus débattus de la religion romaine, c'est-à-dire celui des divinité fonctionnelles (les Sondergötter), mais il le fait de façon exhaustive et avec des catégories d'analyse jamais utilisées jusqu'ici. La grande valeur de cet ouvrage doit être soulignée dès maintenant. 308 pages de recherches pointues, précises, approfondies, qui nous mènent au centre d'un problème ancien : la classification de leurs divinités par les Romains, mais aussi d'un problème plus récent: les interprétations que les savants modernes en ont données. Il faut le dire clairement, il ne s'agit pas d'un livre aisé. La complexité du sujet et la volonté de M.P. de l'explorer en profondeur ont produit un essai dont la lecture ne permet pas de distractions. Pour se laisser conduire par le grand savoir de l'A., il faut s'engager dans une lecture « religieuse », en prenant ce mot dans l'acception primaire de religio, c'est-à-dire «recueillement et scrupule ».

Le livre se compose de deux parties et trois chapitres différents entre eux par l'ampleur et les enjeux. La première partie (correspondant au premier chapitre), est une dissertation qui occupe plus que la moitié du volume. Ce chapitre, dont le titre «Un dio per ogni cosa » est très parlant, analyse toutes les divinités romaines «mineures », c'est-à-dire celles dont le domaine d'action est assez étroit : par exemple des divinités responsables de quelques gestes faits pendant l'accouchement d'une femme et pendant les rapports sexuels, ou des états d'âme particuliers comme les différents aspects de la peur. À ma connaissance, cette partie du livre est l'unique publication existante qui offre un panorama complet de ces divinités assez méconnues. Il s'agit donc d'un travail nécessaire, même si peut-être un peu moins intéressant au niveau intellectuel que les deux autres chapitres du livre. La matière est très vaste et cela explique quelques inadvertances. On remarque par exemple dans la bibliographie l'absence d'une étude à laquelle on se serait attendu, celle de J.-J. AubERT, «La procréation (divinement) assistée dans l'antiquité gréco-romaine », in V. DASEN (éd.), Naissance et petite enfance dans l'Antiquité. Actes du colloque de Fribourg, 28 nov. - $1^{\prime \prime}$ 